\title{
LA SÉCURITÉ CONTRACTUELLE ET LE PRINCIPE DE FAVEUR POUR LE CONTRAT UNE VISION Á PARTIR DU DROIT TRANSNATIONAL *
}

\section{Contractual security and the principle of favor for the contract - A vision from transnational law \\ La seguridad contractual y el principio favor negotii - Una visión desde el derecho transnacional}

Joaquín Emilio Acosta Rodríguez**

José Manuel Gual Acosta***

Recepción: 2 de marzo de 2020. Aceptación: 25 de septiembre de 2020.

DOI: http://dx.doi.org/10.21017/Rev.Repub.2021.v30.a100

\section{RESUME}

Dans cet article, on présente les liens conceptuels entre la sécurité juridique et le principe de faveur pour le contrat. Une analyse de ces liens est proposée afin de reformuler le problème de l'interprétation du contrat aussi bien que de son corollaire, sa validité. En outre, le droit transnational est utilisé pour repenser la force obligatoire du contrat.

Mots-clés: Sécurité juridique, favor negotii, droit transnational.

\section{ABSTRACT}

In this article, we present the conceptual links between legal certainty and the principle of favor for the contract. An analysis of these links is proposed in

* El presente artículo es resultado de los proyectos de investigación: i) “La constitucionalización del derecho de contratos", dentro del grupo de investigación: Derecho, Sociedad e Industrias Creativas de la Escuela de Derecho y Gobierno del Politécnico Grancolombiano; ii) el Grupo de Investigación Estudios en Derecho Privado y Propiedad Intelectual de la Universidad Católica de Colombia.

** Doctor en derecho de los negocios de l'université de Bordeaux - Francia, becado por el Estado francés (becario «Égide»). Máster en derecho privado, Especialista en derecho comercial y abogado. Mail : joaquinacosta2001@yahoo.fr

*** Doctor en derecho civil- responsabilidad, Becado por la Scuola Superiore Sant anna de Pisa - Italia, Docente Investigador Universidad Católica de Colombia. Becario de investigación por la Universidad de Génova y la banca de Italia. Especialista en derecho de los negocios y abogado. Mail: josegual2@gmail.com 
order to reformulate the problem of the interpretation of the contract as well as its corollary, its validity. In addition, the transnational law is used to rethink the binding force of the contract.

Key words: Legal security, favor negotii, transnational law.

\section{RESUMEN}

En este artículo, se presentan los vínculos conceptuales existentes entre la seguridad jurídica y el principio favor negotii. Se propone un análisis de tales conexiones para reformular el problema de la interpretación del contrato, así como su corolario, su validez. Además, se recure al derecho transnacional para repensar la fuerza obligatoria del contrato.

Palabras clave: Seguridad jurídica, favor negotii, derecho transnacional.

\section{INTRODUCTION}

Le principe de la force obligatoire du contrat suppose que l'exécution soit conforme au contenu du contrat (Fauvarque-Cosson, 2008). C'est clair que les contrats peuvent être modifiés par le juge lorsque le législateur lui donne expressément un tel pouvoir. Mais qu'en est-il en l'absence de dispositions législatives ? S'agissant du droit communautaire européen, la sécurité contractuelle est une expression particulière du principe général de sécurité juridique, reconnu comme un des principes généraux du droit communautaire (Lefebvre, 2005). Ainsi cette sécurité est «consubstantiel à la notion même de contrat, ce que reconnaît implicitement la CJCE lorsqu'elle juge qu'en se référant à un accord, l'article $228 \S 1$ al. 2 du traité $\mathrm{CE}$ entend utiliser ce terme dans un sens général, pour designer tout engagement pris par des sujets de droit international et ayant une force obligatoire» (Adida-Canac, 2003). De même, on trouve la nécessité de respecter la loyauté contractuelle sous la forme du principe de coopération loyale posée à l'article 10 du Traité CE. Ainsi la CJCE a fait de «la loyauté des transactions commerciales» (CJCE, 13 déc. 2005) une «raison impérieuse d'intérêt général» (Lefebvre, 2005). Permettant de justifier une entrave à la libre circulation. De la sorte on constate que le principe de loyauté intervient plus largement en tant que principe général du droit communautaire des contrats dans l'ensemble du processus contractuel (AdidaCanac, 2003). Le changement imprévisible des circonstances comporte une exception à la force obligatoire, à travers la notion de force majeure, laquelle englobe divers standards, tels que l'imprévisibilité (CJCE, 13 oct. 1993). Cependant, la sécurité contractuelle ne se limite pas à la question de la révision du contrat pour imprévision. En effet, ce principe aussi produit des 
conséquences, cette fois issues de l'intégration de la situation contractuelle dans l'ordre juridique général. Cette constatation impose de rappeler que la sécurité contractuelle commande le respect de la règle favor negotii. De cette façon se pose la question de l'efficacité adéquate du principe de faveur pour le contrat.

Le principe favor negotii, au service de l'autonomie de la volonté, vise à maintenir le contrat en restreignant la nullité à ce qui est strictement nécessaire pour supprimer la méconnaissance de l'ordre public ou les bonnes mœurs. Il se trouve que celui-ci consiste le plus souvent dans le maintien impératif du contrat partiellement vicié, abstraction faite de toute considération tenant au caractère déterminant de la clause nulle. Ainsi, si un contrat de consommation comporte une clause abusive ou violant un droit essentiel du consommateur, il est évident que le contrat doit être maintenu même si la clause était déterminante aux yeux du professionnel. En effet, la nullité totale du contrat se retournerait contre celui que le droit entendait protéger (en matière de bail commercial renfermant une clause d'exclusivité illicite, Civ. 3e, 31 janv. 2001).

Du droit international des contrats au droit transnational des contrats. «Dès le début du XXe siècle, on a pu observer le passage d'un droit international, fait par les États et pour les États, à un droit transnational - pour reprendre la formule de Philip Jessup, un grand juriste américain - visant tous les échanges à travers les frontières» (Decaux, 2012). Le vœu d'une justice appropriée à leurs besoins peut aussi conduire les opérateurs du commerce international à décider que leur différend sera tranché en application de règles de droit qui ne sont pas celles d'un système juridique déterminé mais des règles «authentiquement internationales» (Hotte, 2007).

Il a pourtant été soutenu que les contrats internationaux n'ont pas une nature différente de celle des contrats internes, la seule spécificité reconnue au contrat international est le «risque juridique» de «l'éventualité d'un dommage causé aux parties ou à l'une d'entre elles par la soumission du contrat à une règle de droit qu'elles ne croyaient pas compétente» (Chaillé de Néré, 2003). Le développement spectaculaire surtout depuis les années 1960, d’un «droit commun des nations» (Fouchard, 1965) composé «de normes d'origine professionnelle, ou de règles coutumières et des principes que des sentences arbitrales révèlent, à moins qu' elles ne les élaborent» apparaît très intéressant: $c^{\prime}$ est dans ce droit commun des nations que les parties trouveront les règles dont elles ont besoin. Ce droit commun l'on désigne comme la nouvelle lex mercatoria, inséparable de l'arbitrage international puisque c'est cette institution qui l'enrichit de nouvelles applications et de nouvelles normes (Goldman, 1979), dont la question de la pénétration de la règle morale dans la vie des affaires laisse aussi ses traces. 
En effet, l'équilibre entre les intérêts particuliers et la morale n'est pas facile á établir (Le Tourneau, 1996). À cette fin, les arbitres du commerce international veillent à ce que les impératifs de prévisibilité et de stabilité contractuelle composent avec les exigences de la bonne foi, constituant ainsi les deux axes principaux du régime arbitral (Taok, 2009). Ces deux axes ne sont pas mutuellement imperméables, la force obligatoire du contrat n'est-elle même pas la traduction du devoir moral de respecter la parole donnée? Ainsi sécurité juridique et moralité sous-tendent l'ensemble des règles régissant le contrat, parmi lesquelles figurent des règles transnationales applicables indépendamment de toute référence á un droit étatique particulier. Ces principes permettent aux arbitres -et par conséquence aux juges aussi- de poser la limites à la force obligatoire de la convention, au nom de la sécurité juridique, dans un esprit de coopération et de loyauté préservant ainsi la morale des affaires.

La nouvelle lex mercatoria se présente comme «un fond commun de règles» qui permet à l'arbitre de régler les contestations qui lui sont soumises sans être lié par la nécessité de respecter un strict ordonnancement juridique (Oppettit, 1998). Ce fonds commun de règles se compose principalement d'usages $d u$ commerce international, de règles transnationales et de règles issues de la jurisprudence arbitrale internationale. Cette conception traduit le pluralisme des sources qui règne dans l'arbitrage commercial international. Cependant, les règles transnationales constituent le cœur de la lex mercatoria par leur nature essentiellement synthétique. Les règles transnationales procèdent de la méthode permettant de dégager les principes généraux du droit (De Béchillon, 1998), lesquels, à un moment ou un autre, se font sentir (Verdross, 1934).

Un autre principe transnational. Le droit transnational des contrats paraît admettre pacifiquement la règle favor negotii: «Une telle alternative peut se présenter dans trois cas de figure: 1.- Lorsque le contrat est sujet à interprétation. 2.- Lorsque la validité du contrat est menacée. 3.- Lorsque l'exécution du contrat est menacée» (Fauvarque-Cosson, 2008, 111).

\section{PROBLEMATIQUE}

Du point de vue méthodologique, cet article est le résultat d'une recherche insérée dans le paradigme herméneutique-interprétatif. En tant que recherche éminemment théorique, l'approche qualitative a eu recours à l'utilisation de méthodes générales telles que l'analyse, la synthèse, l'induction et la déduction et comme outils principalement la révision documentaire.

En ce qui concerne la conceptualisation de la relation entre la sécurité contractuelle et la règle favor negotii et son application, on analyse: 
1. Si le contenu et le champ d'application de la règle favor negotti correspondent à l'idée de sécurité contractuelle, en tant qu'impératif protégeant les attentes des contractants

2. Si, de la même manière, la règle est concordante avec le cadre conceptuel de la sécurité contractuelle, reconnu au niveau transnational.

\section{HYPOTHESE}

Ainsi, les développements qui suivent auront pour objet de montrer que le principe de sécurité contractuelle se révèle être un instrument de protection de la stabilité du contrat, laquelle est au même temps un impératif relatif: la sauvegarde de l'ordre public commande d'anéantir l'efficacité de l'accord -et de cette façon déprotéger les attentes des contractants- si le juge vérifie que celui-ci méconnait des normes impératives. Dans ce contexte, le droit transnational a vocation pour offrir des éléments précieux afin de résoudre une telle problématique.

\section{LA MÉTHODE DE LA RECHERCHE}

Pour mener à bien l'examen herméneutique, le premier exercice de recherche a consisté à rassembler, lire, classer et interpréter des textes originaux ou des fac-similés et des références comparatives occidentaux, qui montrent la portée de la règle favor negotii, à la lumière de l'impératif contemporain de la sécurité contractuelle. En conséquence, il s'agissait dans cette phase d'une enquête sur la collecte, la classification et l'interprétation documentaire comparatiste (analogie / contraste).

Sur la base des textes susmentionnés rassemblés et interprétés dans leur contexte et compte tenu des différentes conceptions existant dans les pays représentatifs de la tradition romaniste, le deuxième exercice de recherche a été mené simultanément, consistant à élaborer un modèle d'analyse original d'observation et de réflexion systématique, dans laquelle chaque environnement correspond à une unité d'analyse divisée en catégories qui répondent à différentes conceptions de la stabilité contractuelle dans l'environnement national correspondant.

Avec le modèle original ainsi élaboré, avec ses unités et catégories d'analyse correspondantes, qui permet de voir le sujet dans une perspective comparative, le troisième exercice de recherche non simultané a été réalisé. Il consistait à l'appliquer au contexte national pour proposer des outils permettant de repenser stabilité contractuelle. 


\section{LES RESULTATS}

On développera ce bilan, en voyant le traitement que le droit transnational donne à ce sujet (A), pour ensuite montrer les perspectives que cette situation offre aux droits de tradition française (B).

\section{A. Le principe de faveur pour le contrat en droit transnational.}

S'agissant de l'interprétation du contrat, les parties doivent être considérées comme des personnes sensées qui ont entendu donner une pleine efficacité à leur contrat (magis ut res valeat quam pereat). Ainsi, si une clause, en raison de sa rédaction ambiguë, peut être interprétée soit de façon à être frappée d'invalidité, soit de façon à être licite, c'est cette dernière interprétation qui doit prévaloir (Rouhette - De Lamberterie - Tallon - Witz, 2003). Cette règle en faveur du plein effet est inscrite dans plusieurs codes civils de l'Union européenne, comme les textes belge, français, luxembourgeois, espagnol, italien, portugais. D'ailleurs, elle est reconnue par la jurisprudence dans la plupart des systèmes de ce continent. Cependant, afin de respecter l'application de l'action collective de l'article 7 (2) de la directive 93/13/CEE sur les clauses abusives dans les contrats conclus avec les consommateurs du 5 avril 1993, l'interprétation en faveur du plein effet ne saurait être retenue car, dans ce cas, il importe de lutter contre les clauses abusives (Rouhette De Lamberterie - Tallon -Witz, 2003). Bref, le principe de faveur pour le contrat impose de préférer la solution qui conserve une efficacité au contrat à celle qui conduirait à son anéantissement.

En ce qui concerne la validité de l'accord, certains droits permettent au juge d'adapter le contrat à ce qu'il aurait dû être selon la volonté des parties en cas d'erreur partagé, Tel est le cas de l'Allemagne, de l'Espagne et du Portugal. Cependant, d'autres systèmes comme le cas de la France, de l'Angleterre et de l'Ecosse refusent de conférer aux juges ce pouvoir (Rouhette - De Lamberterie -Tallon -Witz, 2003). En cas d'erreur peu important, partagé ou non, certains droits autorisent le juge à modifier le contrat pour illustrer cela, il est nécessaire de se rappeler que l'art. 6:230 (2) BW donne au juge néerlandais le pouvoir de modifier le contrat au lieu de l'annuler; en Finlande, Suède, et Norvège le juge dispose du même pouvoir sur le fondement de la disparition des présupposés ou de la clause générale du $\$ 36$ des lois sur les contrats, mais seulement à la demande de la victime de l'erreur; en Autriche le § 872 ABGB permet l'adaptation judiciaire du contrat en cas d'erreur non essentielle ; en droit suisse le Tribunal fédéral applique par analogie l'article 20 II CO pour admettre l'invalidation partielle du contrat si l'erreur ne porte que sur un de ses éléments ; si l'errans n'eut pas conclu du tout le contrat s'il avait connu la réalité, le contrat est invalidé dans son entier (Fauvarque-Cosson, 2008). De 
même, lorsqu'une partie a tiré un profit excessif ou un avantage déloyal de la situation de son cocontractant, certains droits accordent au juge le pouvoir d'adapter le contrat pour supprimer la disproportion qui en résulte, bien que le moyen traditionnel soit la nullité du contrat, comme cela arrive en Autriche, France, Luxembourg, Portugal, Pays Bas, Danemark (Rouhette - De Lamberterie -Tallon -Witz, 2003). Notamment au travers de la laesio enormis. Le droit anglais, au contraire, dispose le remplacement légal des clauses invalides du contrat. Par le biais de l'interprétation, le juge anglais procède à un complétement du contrat, afin de protéger son efficacité. La méthode employée est le «business efficacy test»: les parties sont censées souhaiter les engagements nécessaires pour la pleine exécution économique du contrat (business efficacy) (FauvarqueCosson, 2008). Tandis que certains droits comme le suisse retiennent que, lorsqu'une clause d'invalidité touche une partie non-essentielle de l'accord la convention peut être maintenue en annulant la partie viciée. Certes, cette solution n'est pas générale: 1 'annulation atteint en principe tout le contrat tel est le cas des droits nordiques. Cependant, certains systèmes connaissent des solutions qui permettent de sauver le contrat vicié de nullité. La technique principale est celle de la «conversion par réduction». Elle est notamment connue en droit italien, allemand, suisse, néerlandais et français (Fauvarque-Cosson, 2008). Quant à l'exécution du contrat, le principe favor negotii se traduit par la tendance transnationale $\mathrm{d}^{\prime}$ admettre restrictivement la résolution du contrat inexécuté: celle-ci n'est admise qu'en cas d'inexécution essentielle du contrat, (Fauvarque-Cosson, 2008 ; Rouhette - De Lamberterie - Tallon - Witz, 2003)[1].

Droit international et communautaire. La Convention de Vienne ne traite ni de la validité ni de l'interprétation du contrat de vente (Fauvarque-Cosson, 2008); cependant, elle limite la résolution aux hypothèses de contravention essentielle (arts 49, 64, 72 y 73). La Cour de justice des Communautés européennes emploie la notion d' «effet utile» comme méthode d'interprétation afin de permettre au droit communautaire de «déployer la plénitude de ses effets» (Debard, Le Baut-Ferrarèse, Nourrisat, 2002). Ce principe de faveur se retrouve plus directement au sein notamment de la directive 93/13/CEE du 5 avril 1993 modifié par la directive 2011/83/UE, concernant les clauses abusives dans les contrats conclus avec les consommateurs. Des telles clauses seront réputées non écrites et le contrat pourra recevoir exécution.

Codifications savantes. L'article 4.5 des Principes Unidroit consacre la règle privilégiant une interprétation utile du contrat. Ainsi «Les clauses d'un contrat s'interprètent dans le sens avec lequel elles peuvent toutes avoir quelque effet, plutôt que dans le sens avec lequel certaines n'en auraient aucun». Sans qu'il fasse l'objet d'un article au sein des Principes du droit européen du contrat, le principe favor negotii connaît de nombreuses applications. En matière d'interprétation on trouve l'article 5:106 relatif à l' «Interprétation utile». Ainsi, 
«on doit préférer l'interprétation qui rendrait les clauses du contrat licites et de quelque effet, plutôt que celle qui les rendrait illicites ou de nul effet». L'avant-projet de réforme du droit des obligations et de la prescription consacre implicitement le principe de faveur pour le contrat en matière d'interprétation à l'article 1139-1: «Lorsqu' une clause est susceptible de deux sens, on doit plutôt l'entendre dans celui avec lequel elle peut avoir quelque effet, que dans le sens avec lequel elle n'en pourrait produire aucun». De façon analogue, l'avantprojet de Code européen des contrats énonce une règle de préférence pour l'interprétation utile du contrat. Selon son article 40 (2): «Dans le doute, le contrat ou les clauses séparées doivent être interprétés dans un sens qui leur confère quelque effet plutôt que dans un sens qui les empêcherait d'en produire».

Quant à la validité de la convention, les Principes Unidroit (PCCI) connaissent plusieurs applications du principe de favor negotii. Tel est le cas des hypothèses de maintien du contrat en dépit de l'erreur commise (art. 3.13) ou d'adaptation du contrat en cas d'avantage excessif (art. 3.10). Aussi la nullité partielle sera préférée chaque fois qu'il ne sera pas déraisonnable de maintenir le contrat en son entier (art. 3.16). La confirmation est de même admise par cette élaboration (art.3.12). Diverses dispositions des Principes du droit européen du contrat (PDEC) admettent la modification de l'accord afin d'éviter sa nullité (art. 4:105 ou 4:109). L'esprit de ces solutions est «qu'il convient de sauver le contrat lorsque l'une des parties y a intérêt» (Fauvarque-Cosson, 2008). Encore un anéantissement partiel est préféré à un anéantissement total. Tel est le cas lorsque sont seules sanctionnées les clauses affectées par l'erreur, le dol, la contrainte ou le profit excessif. Ce n'est que s'il est déraisonnable de maintenir la totalité de l'accord que celui-ci devra être entièrement annulé (art. 4:116). Le même mécanisme de nullité partielle est appliqué au contrat en cas d'illicéité (art. 15:103). Quant à l'exécution du contrat, les articles 6:104 à 6:107 «posent des règles qui peuvent servir à 'sauver' le contrat dans les cas où cela semble raisonnable» (Rouhette, De Lamberterie, Tallon, Witz, 2003). On a déjà vu un exemple de cette conception lorsqu'on a exploré le régime du prix déraisonnable (art. 6:105). S'agissant du droit de résolution du contrat par inexécution, celui-ci ne peut s'exercer que s'il y a «inexécution essentielle de la part du cocontractant» (Arts. 9:301, 8:103 et 9:302). L'avant-projet de Code européen des contrats (CEC) retient la possibilité d'une nullité partielle en admettant la validité du contrat «dans sa partie restante, pourvu que cette dernière possède une consistance et une validité autonomes et réalise de manière raisonnable le but poursuivi par les parties» (art. 143). Cette codification connaît également techniques alternatives à la nullité du contrat (art. 144), telle que la conversion par réduction (arts. 145,143 et 40), si bien les parties peuvent l'exclure expressément (art. 145). On trouve également la régularisation du contrat (art. 143). La confirmation 
du contrat nul est également admise (art. 149). L'avant-projet de réforme du droit des obligations et de la prescription fait prévaloir la nullité partielle lorsque la clause entachée de nullité n'a pas «constitué un élément déterminant de l'engagement des parties ou de l'une d'elles» (art. 1130-2). Au titre des alternatives à la nullité, ce projet connaît la régularisation du contrat (art. 1133) comme sa confirmation (art. 1129-4).

S'agissant enfin de l'exécution de l'accord, le principe de faveur se retrouve au sein des Principes Unidroit dans les règles qui prévoient des mécanismes de substitution lorsque le prix fixé est déraisonnable ou que le mode de fixation choisi est défaillant (art. 5.1.7). Quant à la résolution de l'accord pour inexécution, les Principes Unidroit ne l'admettent que si l'inexécution est essentielle (Art. 7.3.1). Les règles retenues par les PDEC rappellent celles des Principes Unidroit. En effet, si le prix ou un autre élément du contrat est fixé de manière manifestement déraisonnable il et remplacé par un élément raisonnable (art. 6:105). De même, lorsque l'inexécution contractuelle est vérifiée, la validité de l'accord, totale ou partielle, est préférée (art. 8:103). Ainsi, la résolution ne joue qu'en cas d'inexécution essentielle (Art. 9:301). Cependant, même lorsqu'il y a inexécution essentielle le principe de faveur peut encore jouer, non sur le domaine de la sanction mais sur son étendue. En effet, il n'y a pas lieu de résoudre tout le contrat si celui-ci doit être exécuté «par tranches» (art. 9:302). Dans l'avant-projet de Code européen des contrats seules quelques applications ponctuelles du principe de favor negotii peuvent être citées. Ainsi, la carence des parties quant à la détermination du lieu d'exécution est palliée par les règles de l'article 82. De façon analogue aux autres projets évoqués, la résolution contractuelle n'est admise qu'en cas d'inexécution «d'importance notable» (art. 114). Au contraire, au sein de l'avantprojet de réforme du droit des obligations et de la prescription, le principe de faveur pour le contrat paraît en retrait, du moins s'agissant de la résolution du contrat. En effet, l'article 1158 offre au créancier victime de l'inexécution, même de l'exécution imparfaite, l'alternative entre l'exécution forcée, la résolution et la demande de dommages et intérêts. Cependant, il résulte un tempérament de l'article 1158-1, car cette disposition précise $\mathrm{qu}^{\prime}$ «[i]l est loisible au débiteur de contester en justice la décision du créancier en alléguant que le manquement qui lui est imputé ne justifie pas la résolution du contrat». De même chacun de ces projets retient qu'avant toute résiliation, ou pour ceux qui l'admettent, toute modification judicaire de la convention, les parties sont tenues de renégocier le contrat afin de le sauver (art. 6.2.3 PCCI, art. 6:111 PDEC; arts. 97 et 157 CEC; art. 1135-2 et 3 avant-projet de réforme du droit des obligations et de la prescription).

À manière de synthèse, les Principes contractuels communs proposent le texte suivant: 


\section{«Article 0:204: Faveur pour le contrat}

Lorsque le contrat est sujet à interprétation, ou lorsque sa validité ou son exécution est menacée, l'efficacité du contrat doit être préférée si son anéantissement nuit aux intérêts légitimes de l'un des contractants».

\section{B. Perspectives pour les droits de tradition française.}

Droit français. En matière d'interprétation du contrat, ce système accueille ouvertement la règle favor negotii dans l'article 1157 du Code Napoléon, selon lequel «[1]orsqu'une clause est susceptible de deux sens, on doit plutôt l'entendre dans celui avec lequel elle peut avoir quelque effet, que dans le sens avec lequel elle n'en pourrait produire aucun». Ainsi, on peut raisonnablement présumer que, lorsque deux sens sont possibles, la commune intention des parties a plutôt été de retenir le sens qui confère une certaine efficacité á l'acte que celui qui conduit au résultat contraire (Cas. Civ. 3e, 19 déc. 1983). Or, cette disposition fait partie de la section V dudit Code, dédiée à «l'interprétation des conventions». En droit, l'interprétation est l'opération qui consiste à discerner le véritable sens d'un texte obscur (Cornu, 2003). On a souvent souligné l'analogie qui existerait entre l'interprétation des normes juridiques et l'interprétation des contrats. En effet, il s'agirait dans les deux hypothèses de préciser le sens et éventuellement de combler les lacunes des textes juridiques aussi bien que contractuels. Certes, cette remarque ne permet pas d'ignorer la différence profonde qui sépare ces deux catégories de textes: si la loi est l'expression de la volonté générale, le contrat n'est que l'expression de la volonté des parties. Ainsi, tandis que l'interprétation d'une convention est une question de fait, casuistique, l'interprétation des normes juridiques -par des raisons d'égalité et de sécurité juridique- de la part des juges doit être développée dans un cadre d'uniformité (Terre - Simler - Lequette, 2013). Cependant, la fondamentalisation du droit des contrats impose aussi dans ce domaine de repenser la conception traditionnelle. En effet, aujourd'hui le juge des contrats aborde d'une manière différente -plus humanisante et plus équilibrée- le contrat et son interprétation. Les droits fondamentaux sont utilisés comme norme de référence pour interpréter le contrat: «C'est ainsi que l'impact des droits fondamentaux sur le contrat est susceptible d'entraîner une réflexion bien plus large sur le contrat et les principes directeurs qui le gouvernent. L'influence des droits fondamentaux sur l'interprétation suppose de se positionner sur une approche nouvelle du contrat, de se questionner par exemple sur une redéfinition de l'autonomie de la volonté, sur la source des obligations découlant du contrat, sur l'importance que l'on souhaite accorder à l'interventionnisme du juge» (Caumes 2010). Ainsi cette évolution inciterait à se placer à un point de vue plus élevé, celui de la justice et en concourant à définir ce qui est dû (Jacques, 2003). 
De cette façon, cette nouvelle conception de l'interprétation pourrait être de nature à conférer au juge des pouvoirs très importants, notamment celui de modifier le contrat pour le rééquilibrer, toujours au nom de l'interpréter. Cependant, la tradition dénonce qu'une telle conception irait directement à l'encontre de la sécurité juridique, sans laquelle le contrat, qui est d'abord un «acte de prévision sur l'avenir», perdrait sa nature (Lécuyer, 1999). Bref, le pouvoir du juge français pour interpréter les conventions ne va pas jusqu'au point de dénaturer les conventions. La Cour de cassation se reconnaît, depuis un arrêt du 15 avril 1872, le droit de censurer les décisions des juges du fond lorsque les termes employés par les parties sont clairs et précis et que les obligations résultant du contrat ont été dénaturées par les magistrats (Capitant - Terre - Lequette, 2000). La Cour de cassation se donne ainsi les moyens d'empêcher les juges du fond de refaire le contrat en équité sous prétexte de l'interpréter (Boré, 1972) (Voulet, 1971). Cependant, malgré la préférence traditionnellement donnée à l'impératif de stabilité, l'équité joue un rôle croissant dans le droit français des contrats (Terre - Simler - Lequette, 2013). En effet, si la jurisprudence ne se reconnaît pas le pouvoir de réécrire en son nom le contrat, elle puise de plus en plus dans celle-ci, celui d'adjoindre au contrat des conséquences que les parties n'avaient pas voulues. Les articles 1134 et 1135 sont les textes invoqués pour justifier cette nouvelle conception de l'interprétation des contrats. De cette façon à côté de l'interprétation «subjective», directement inspirée par la théorie de l'autonomie de la volonté et qui fait du juge le serviteur de la volonté des parties, émerge l'interprétation «objective», par laquelle le juge des contrats interprète l'accord, non en s'épuisant à rechercher une commune intention inexistante ou hypothétique des parties, mais en faisant appel à la bonne foi, à l'équité contractuelle et aux usages des affaires (Paclot, 1985), (Ivainer, 1981), (López, 1968), (Rieg, 1961) (Marty, 1949) (Déreux, 1905). Alors que pour la doctrine classique, ces notions constituent un simple prolongement de la volonté des contractants, qui sont censés s'y être référés tacitement, elles acquièrent avec la doctrine post-moderne une existence propre. Plutôt que d'essayer de découvrir la commune intention des parties, on recherchera ce qui est juste, ce qui est habituellement pratiqué (Terre - Simler - Lequette, 2013). Bref, plutôt que de rechercher ce que les parties auraient pu vouloir si elles s'étaient posé la question, le juge s'érige en ministre d'équité (Cas. Civ. 2e, déc. 1947). Celuici fixe le contenu des obligations de chacune des parties en s'inspirant du sentiment de justice.

Favor negotti et validité de l'accord. Quant à la validité du contrat, le Code Napoléon ne comporte aucune disposition générale relative à la question de l'étendue de la nullité. Ses rédacteurs ne se sont intéressés qu'aux conditions impossibles, illicites ou immorales dont un acte peut être affecté et ont proposé des solutions diamétralement opposées selon que de telles conditions figurent 
dans les actes à titre gratuit ou dans les conventions à titre onéreux. Dans le premier cas, l'article 900 du Code civil dispose que les conditions viciées «seront réputées non écrites», alors que, dans le second, l'article 1172 décide que la condition nulle «rend la convention qui en dépend». C'est pourquoi la jurisprudence française a entrepris une audacieuse et habile œuvre d'interprétation. Ainsi, si bien traditionnellement le principe a été celui de la nullité intégrale du contrat (Cas. Com. 20 juill. 1983 et Cas. Civ. $1^{\text {re }}, 7$ mai 1963) ce système admet la nullité partielle de l'accord. En effet, de plus en plus la «législation interventionniste et pointilliste» post-moderne (Terre Simler - Lequette, 2013) dispose que telle clause seulement ou telle partie $\mathrm{d}^{\prime}$ un acte soit nulle et que l'on puisse concevoir que cet acte continue de produire ses effets pour le surplus. Comme aboutissement de l'évolution de la jurisprudence aussi bien que de la législation française, la doctrine constate que la détermination de l'étendue de la nullité contractuelle correspond à une recherche de l'intention des parties, si elle constituait à leurs yeux la «cause» ou la «condition impulsive et déterminante»: dans ce cas, l'acte tout entier est nul ${ }^{1}$. Si au contrarie la clause revêtait un caractère secondaire, de sorte que si les parties avaient su que cette clause était nulle, elles auraient vraisemblablement contracté néanmoins, l'acte mérite d'être maintenu (Cas. Civ. 3e, 13 févr. 1969). Cette évolution illustre la vigueur persistante du principe d'autonomie de la volonté. Cependant, cela ne comporte pas d'ériger ce principe en dogme absolue, ici comme ailleurs. Il peut arriver que l'ordre public impose des solutions s'écartant de celles qui résulteraient de l'application du critère subjectif de la recherche des intentions. En effet, la nullité totale du contrat se retournerait contre celui que le droit entendait protéger. Les exemples de cette nature, dans le contexte de la législation instituant des mesures d'ordre public de protection, sont nombreux. La formule figurant abondamment dans la normativité interventionniste contemporaine selon laquelle telle clause est «réputée non écrite» est habituellement révélatrice de la volonté du droit de simplement supprimer la clause contraire, le contrat amputé étant maintenu pour le surplus (Ghestin - Loiseau, - Serinet, 2013). À titre d'exemple on trouve l'article L. 132-1 du Code de la consommation lequel dispose que les clauses abusives sont réputées non écrites. En effet, l'éradication de la clause apparaît comme la meilleure protection du consommateur qui n'a généralement pas intérêt à la disparition du contrat» (Picod, 2004).

À côté de la mutilation de l'acte, on trouve un autre procédé de la nullité partielle: la réduction du quantum excessif. Moyennant celui-ci un élément de

1 Cas. Civ. 3e, 24 juin 1971, JCP 1972.II.17191, note J. GHESTIN 
l'acte tel que sa durée ou son prix est réduit. Il faut remarquer qu'il n'y a pas suppression: le montant d'une obligation est simplement remplacé par un autre, car le premier était excessif (Bakouche, 2005). De même on repère la conversion par réduction. Il s'agit d'une technique juridique qui marque le passage d'une situation à une autre. -conversion de la séparation de corps en divorce- ou d'un acte juridique à un autre -conversion du métayage en fermage (Terré Simler - Lequette, 2013). Certes, la question se pose de savoir sur la portée de cette innovation: véritable novation? (Couret, 1981). En matière de nullités cette institution comporte des solutions novatrices, car la conversion désormais permet de faire produire des effets à un acte traditionnellement nul, impliquant une fonction salvatrice: $l^{\prime}$ acte nul qui cependant remplit les conditions requises pour la validité d'un autre acte, produit un résultat conforme à l'intention des parties, car un tel acte maintien sa validité. Cette technique de sauvetage des actes juridiques n'est admissible que dans le respect de l'intention probable des parties (Boujeka, 2002).

Favor negotti et exécution de l'accord. Quant à l'exécution, le principe de faveur pour le contrat commande au juge de ne pas prononcer la résolution qu'en cas d'inexécution grave (Capitant - Terre - Lequette, 2000). Tel comme on a déjà vérifié, selon jurisprudence relativement récente la gravité du manquement peut justifier la résolution unilatérale du contrat, aux risques et périls néanmoins de l'auteur de la résolution (Cas. Civ. 1e, 20 févr. 2001).

\section{CONCLUSION}

On vérifie que le droit transnational reste sensible aux élaborations doctrinales suivant l'équité contractuelle et la justice commutative, même en matière de sécurité contractuelle. En fait on confirme les liens indissociables existants entre liberté et sécurité contractuelles. De même, la sécurité contractuelle contient aussi le principe de faveur pour le contrat. Cela comporte admettre qu'il peut être préjudiciable, notamment par des raisons économiques, de procurer l'exécution originale prévue par les parties lorsque le créancier pourrait obtenir aisément auprès d'un autre sujet la prestation non exécutée par le véritable débiteur. Certes, on doit procurer l'accomplissement strict du principe favor negotii dans les hypothèses où c'est un besoin essentiel ou déterminante du contrat qui est compromis. De même, il prévaut la sauvegarde, du moins partielle du contrat chaque fois que l'anéantissement de l'accord comporte nuisance aux intérêts légitimes des contractants loyaux et honnêtes. Cela ouvre la porte à des nouvelles perspectives en matière d'interprétation des conventions, aussi bien que pour le régime des nullités contractuelles, à côté de la remise en cause du régime traditionnel de l'exécution de l'accord. 


\section{PRINCIPALES ABRÉVIATIONS}

$\begin{array}{ll}\text { ABGB } & \text { Allgemeines Bürgerliches Gesetzbuch (Code civil autrich } \\ \text { Aff. } & \text { Affaire } \\ \text { BW } & \text { Burgerlijk Wetboek (Code civil néerlandais) } \\ \text { Cas. Civ. 1 }{ }^{\text {re }} & \text { Première Chambre Civil de la Cour de Cassation } \\ \text { Cas. Civ. 2 } & \text { Deuxième Chambre Civil de la Cour de Cassation fre } \\ \text { Cas. Civ. } 3^{\text {e }} & \text { Troisième Chambre Civil de la Cour de Cassation } \\ \text { Cas. Com. } & \text { Chambre Commercial de la Cour de Cassation fra } \\ \text { CE } & \text { Selon le contexte: Conseil d'État français, Com } \\ & \text { Européenne ou Conseil de l'Europe } \\ \text { CEE } & \text { Communauté Économique Européenne } \\ \text { CJCE } & \text { Cour de justice des Communautés européennes } \\ \text { CO } & \text { Code suisse des obligations du 30 mars 1911 } \\ \text { JCP } & \text { Juris-classeur périodique (La Semaine Juridique) } \\ \text { JDI } & \text { Journal du droit international (Clunet) } \\ \text { LGDJ } & \text { Librairie Générale de Droit et de Jurisprudence } \\ \text { PCCI } & \text { Principes Unidroit } \\ \text { PDEC } & \text { Principes du Droit Européen des Contrats } \\ \text { PUAM } & \text { Presses Universitaires d'Aix-Marseille } \\ \text { PUF } & \text { Presses Universitaires de France } \\ \text { SLC } & \text { Société de Législation Comparée } \\ \text { UE } & \text { Union Européenne }\end{array}$

\section{RÉFÉRENCES}

\section{La doctrine}

Adida-Canac, H. (2003). Contribution à l'étude du droit communautaire des obligations, Atelier National de Reproduction des Thèses, vol. 2.

Bakouche, D. (2005). L'excès en droit civil, thèse Paris II.

Boré, J. (1972). «Un centenaire: le contrôle par la Cour de cassation de la dénaturation des actes», RTD civ., 249 - 306.

Boujeka, A. (2002). «La conversion par réduction: contribution à l'étude des nullités des actes juridiques formels », RTD com. 223 - 244.

Capitant, H. - Terré, F. - Lequette, Y. (2000). Les grands arrêts de la jurisprudence civile, obligations -contrats spéciaux, Paris, t.2. 
Caumes, C. (2010) «L'interprétation du contrat au regard des droits fondamentaux», n 349 ; thèse disponible à l'adresse internet www.theses.fr/2010AVIG2021.pdf

Chaillé de Néré, S. (2003) Les difficultés d'exécution du contrat en droit international privé, Aix, PUAM.

Cornu, G. (2003) Vocabulaire juridique, Association Henri Capitant, Paris, PUF.

Couret, A. (1981) «La notion juridique de conversion », in Mélanges Vigreux P, Toulouse, p. $219-260$.

De Béchillon, M. (1998), «La notion de principe général en droit privé», Aix - Marseille, PUAM.

Debard, T. - Le Baut-Ferrarèse, B. Nourrisat, C. (2002) Dictionnaire du droit de l'Union européenne, Paris, Ellipses.

Decaux, E. (2012) Du droit international au transnational», article disponible à l'adresse internet http:/ / www.revues-plurielles.org/_uploads/pdf/47/252/p018_20.pdf

Déreux, G (1905). De l'interprétation des actes juridiques privés, TD, Paris.

Fouchard, Ph. (1965), L'arbitrage commercial international, Paris, Dalloz.

Ghestin, J. - Loiseau, G - Serinet Y.-M. (2013) - La formation du contrat, l'objet et la cause, les nullités, 4e éd., V. II, Paris LGDJ.

Goldman, B. (1979), «La lex mercatoria dans les contrats et l'arbitrage internationaux: réalité et perspectives» JDI, P. $475, \mathrm{n}^{\circ} 21, \mathrm{p} .487$ et ss.

Hotte, S. (2007) La rupture du contrat international-Contribution à l'étude du droit transnational des contrats, Paris, Defrénois.

Ivainer, Th., (1981) «La lettre et l'esprit de la loi des parties», JCP,I.3023, 247 et ss.

Jacques, Ph. (2003), «Regards sur l'article 1135 du Code civil», thèse Paris XII.

Le Tourneau, Ph. (1996), «Existe-t-il une morale des affaires ?» in La morale et le droit des affaires, Actes du colloque organisé à l'Université des Sciences Sociales de Toulouse le 12 mai 1995, Montchrestien.

Lécuyer, H. (1999), «Le contrat, acte de prévision», in Mélanges F. Terré, Paris, Dalloz, p. 643 et ss.

Lefebvre, F. (2005), Mémento Pratique - Union européenne 2006-2007. Paris.

López, J. (1968), Les systèmes d'interprétation des contrats, thèse Ph.d, dactyl. Paris II. 
Marty, G. (1949), Rôle du juge dans l'interprétation des contrats, in Trav. Ass. H. Capitant, t. v, p. $84 \mathrm{~s}$.

Oppettit, B. (1998), Théorie de l'arbitrage, Paris, PUF.

Paclot, Y (1988). Recherches sur l'interprétation juridique», TD, Paris II.

Picod, Y. (2004), Nullite, in Rép. Dalloz, Paris.

Fauvarque-Cosson, B., Mazeaud, D., Racine J.-B. et Wicker, G. (2008), Principes contractuels communs, Projet de Cadre commun de référence, SLC, 2008, p. 111.

Rieg, A, (1961), Le rôle de la volonté dans l'acte juridique en droit civil français et allemand, Paris, LGDJ.

Rouhette, G. - De Lamberterie, I. - Tallon, D. - Witz, C. (2003), Principes du droiteuropéen du contrat, Paris, SLC.

Taok, M. (2009) La résolution des contrats dans l'arbitrage commercial international, Paris, L.G.D.J.

Terré F. Simler p. Lequette, Y. (2013), Droit civil. Les obligations, 11éd., Paris, Dalloz.

Verdross, A. (1934) «Les principes généraux du droit comme source du droit des gens» in Mélanges Gény, t. III, p. 383 et ss.

Voulet J, (1971), Le grief de dénaturation, JCP.I, p. 2410.

\section{Jurisprudence}

\section{Cour de justice de l'Union européenne.}

CJCE, 13 déc. 2005, SEVIC Systems AG, Aff. C-411/03, Rec. 2005 p. I-10805.

CJCE, 13 oct. 1993, An Bord Bainne Co-operative Ltd. et Compagnie Interagra S.A. v. Intervention Board for Agricultural Produce, Aff. C-124/92, Rec. 1993 page I-05061.

\section{France}

Cas. Civ. 1re, 20 févr. 2001, Bull. civ. I, $\mathrm{n}^{\circ} 40$

Cas. Civ. 3e, 31 janv. 2001, JCP 2001.I.354 nº 1, obs. Serinet

Cas. Com. 20 juill. 1983, D. 1984, 422, note J.-L. Aubert

Cas. Civ. 3e, 19 déc. 1983, Bull. civ. III, nº 267.

Cas. Civ. 3e, 13 févr. 1969, JCP 1969.II.15942, 2e esp., note J.-Ph. Lévy

Cas. Civ. 1re, 7 mai 1963, Bull. civ. I, nº 246.

Cas. Civ. 2e, déc. 1947, Gaz. Pal. 1948.1, 36 